\title{
An Agricultural Tri-dimensional Pollution Data Management Platform Based on DNDC Model
}

\author{
Lihua Jiang ${ }^{1,2}$, Wensheng Wang ${ }^{1,2}$, Xiaorong Yang ${ }^{1,2}$, \\ Nengfu Xie ${ }^{1,2}$, and Youping Cheng ${ }^{3}$ \\ ${ }^{1}$ Agriculture Information Institute, Chinese Academy of Agriculture Sciences, \\ Beijing, 100081, China \\ ${ }^{2}$ Key Laboratory of Digital Agricultural Early-warning Technology, \\ Agriculture Information Institute, Chinese Academy of Agriculture Sciences, Beijing, 100081 \\ ${ }^{3}$ Agriculture Bureau, Huailai County, Hebei Province, 075400, China \\ \{jianglh, wangwsh, yxr, nf.xie, youping\} @caas.net.cn
}

\begin{abstract}
DNDC is a computer simulation model of carbon and nitrogen biogeochemistry in agro-ecosystems. It is used in agricultural tri-dimensional pollution control widely. Learning from abroad advanced technologies and research methods, we have developed an agricultural tri-dimensional pollution data submission and management platform based on DNDC model. The platform is very important for sharing and building our agricultural carbon and nitrogen chain database.
\end{abstract}

Keywords: DNDC, United Storage, Format Conversion.

\section{Introduction}

DNDC (DeNitrification-DeComposition) is a computer simulation model of carbon and nitrogen biogeochemistry in agro-ecosystems. The model can be used for predicting crop growth, soil temperature and moisture regimes, soil carbon dynamics, nitrogen leaching, and emissions of trace gases including nitrous oxide $\left(\mathrm{N}_{2} \mathrm{O}\right)$, nitric oxide (NO), dinitrogen $\left(\mathrm{N}_{2}\right)$, ammonia $\left(\mathrm{NH}_{3}\right)$, methane $\left(\mathrm{CH}_{4}\right)$ and carbon dioxide $\left(\mathrm{CO}_{2}\right)$. Studying on carbon and nitrogen chain in agricultural tri-dimensional pollution [1] and blocking carbon and nitrogen pollution sources is very important for prevention and treatment agricultural carbon and nitrogen pollution [2]. Development DNDC [3] model needed data management system can undoubtedly provides important scientific support for our country agriculture carbon and nitrogen emission reduction [4] database construction and data sharing.

Study and develop a data submission, management and supporting DNDC model platform for every level user in agriculture tri-dimensional [5] preventive treatment centre. Data submission and management module provides data management interface for administrators and is in favor of adjusting agriculture tri-dimensional pollution data. Assistant DNDC [6] model module can read out data from DNDC database for users and create txt files and also imports DNDC model results to database according to user' willing. 


\section{Platform Main Functions}

This platform is made up of distributed data submission and management module, submitted data centralized management module and assistant DNDC module. The functions of platform are shown in figure 1 .

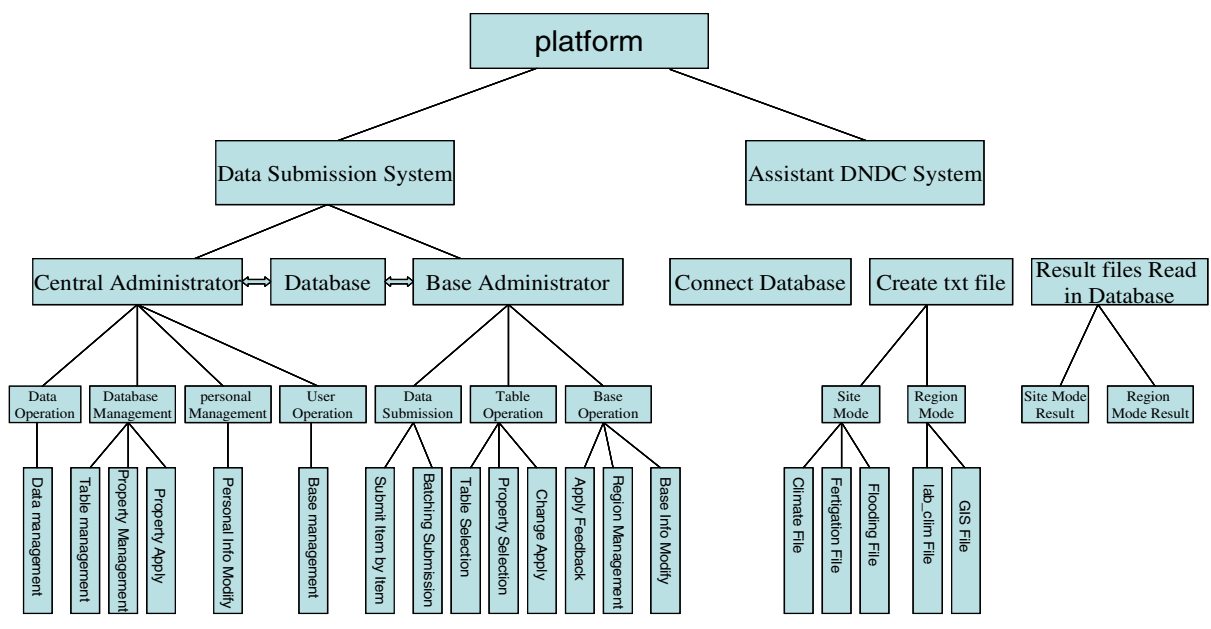

Fig. 1. Platform main functions

\subsection{Distributed Data Submission and Management Module}

Distributed data submission and management module provides several function interfaces for users and base administrator can realize heterogeneous data submission. Base administrator can submit the collected agricultural tri-dimensional data to central database by using function interface in favor of centralized and unified management. Central administrator can manage all base submitted information including modifying and deleting the information. In addition, base administrator can self-determine the submitted tables and corresponding attributes of tables and make up personal operation interface. The platform realizes personal management.

\subsection{Submitted Data Centralized Management}

Platform can realize central management of submitted data from bases and provides functional interface in which central administrator can modify construction of database directly. It is very convenient and visual for users to operate database. The operation of database construction includes adding and deleting tables and adding and deleting attributes of tables. 


\subsection{Assistant DNDC Module}

Functions of the module is shown in figure 2 including database connecting, data format conversion from database to DNDC model, storage of results from DNDC stimulation model to database. DNDC model sometimes will use some text files for example climate files, fertigation files, flooding files in site mode and initial data files in region mode and these files can created in assistant DNDC module. User can read result files from DNDC model to database in the platform. Within the power of privilege, user can select different formats result files in different modes and store them in corresponding database.

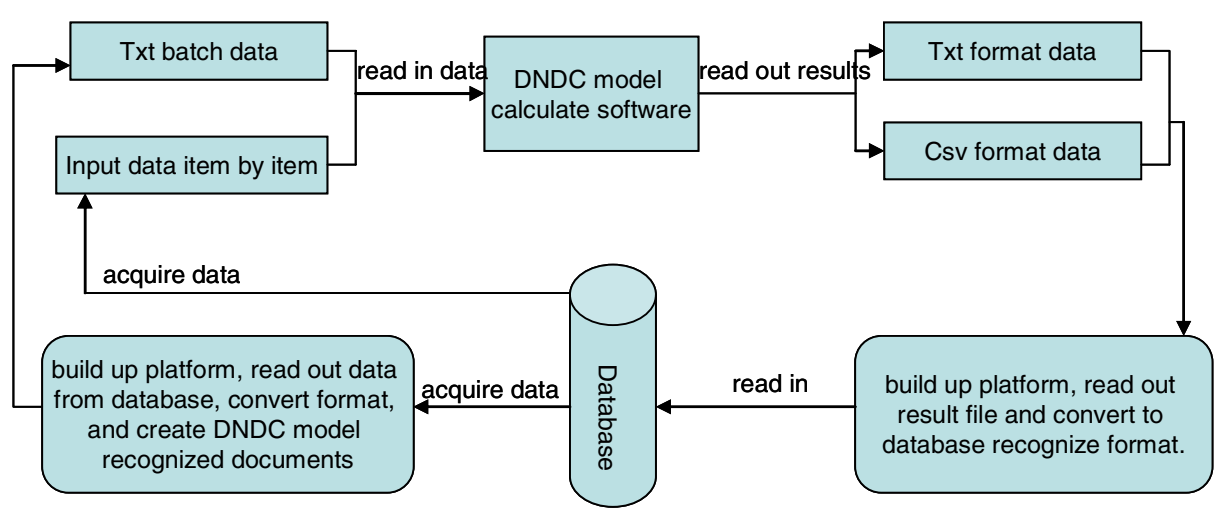

Fig. 2. Main functions logic diagram of assistant DNDC module

\section{Key Technologies}

In developing process, we solve the key technology problems including distributed heterogeneous data submission, data submission item by item or in batch, format conversion from database to DNDC model, storage of DNDC model results, function management mechanism based on role and so on.

\subsection{Distributed Heterogeneous Data Submission Technology}

In distributed data submission process, the tables submitted by different bases are different. If in designing process, every base is shown all the tables, the system is not well targeted and not convenient for users. But if every base corresponding table is designed in advance, it is also not perfect, because it is possible to change the submitted data tables. The fields in tables are in the same reasons. Aiming at above problems, the platform provides table views and user personal control of submission interface fields display function. At first, users can select needed tables by random in platform and then navigation bar will embody users' personal choice and provide pointed interface. In the next 
place, users can select corresponding field attributes of tables in function interface and make up personal submission interface. It is convenient for different users to operate.

\subsection{Data Submission Item by Item or in Batch Technology}

Distributed data submission and management module mainly realizes different bases and different information submission function. So submission function is the key and difficult problem of the platform. Because the quantity of bases using the platform is not limited and data bulk submitted by bases is not limited, if system only provides submission item by item function, when submitted data bulk is so large, system will waste a lot of time. So system also provides submission in batch function. In submission data item by item, formats of submitted data must be controlled seriously when base administrator submits data to central databases. In submission item by item interface, platform provides description of submitted attributes data format and clear clue of input error. User can realize submit a small quantity of unpacked data correctly. When user needs to submit a mass of packed data, data submitting in batch module is needed and shows superiority fully. The system provides two different batching submission means: (1) Import from Excel files to database. If user has already stored collected data in definite format in Excel files and can use the batching submission interface to import them to central database quickly after mapping. It can save a lot of importing item by item time. (2) Import data from local database to central database. In many cases, user stores collected data in local database in favor of partial management. At the moment, user can use batching submission module to copy local data to central database quickly.

\subsection{Format Conversion Technology from Database to DNDC Model}

The central database stores data from every base. Administrator stores them in fixed format to many stables in database. When DNDC model is used to stimulate agricultural tri-dimensional pollution preventive treatment, data in database is needed and a lot of them need to be adopted in fixed text document format. Formats of the data in database should be converted to format which DNDC model can recognize and use. Format conversion is a dynamic form. Platform shows all choices possibly used in text files, every table name relevant to DNDC model in database and all fields name of the tables. When user uses the platform, he can decide items in text file and the table and fields in database.

\subsection{Storage DNDC Model Results to Database Technology}

User uses DNDC model to stimulate and get some results. According to different stimulation in different modes, the result files have two formats: txt and csv. The format of result data in above two formats result files is the same and assistant DNDC module can read out and show data items of result files. If user wants read data item in result files in some table in database, he can dynamically select data item in choice box. In the process of reading in, user can decide how to map data items into the fields in tables or whether read data items to correspond tables in database. And txt result file data can be 
read in five tables in the database and user can decide whether read in tables or which tables should be read in. Csv result file data can be read in one table in database. When user selects fields corresponding to data item, he can import all data to database. When the above two types of files are imported to database, the user' name is stored in database automatically in order that central administrator can adjust database management.

\subsection{System Function Management Mechanism Based on Role}

The system has three user roles: central administrator, base administrator and DNDC user. Base administrator collects local base data and submits to platform. Central administrator has all management privilege and can limit base administrator' management privilege. When DNDC user uses DNDC model, central administrator will give privilege to use assistant DNDC model. DNDC user can be base administrator and central administrator and also other privileged user.

\section{Technology Innovation}

\subsection{Distributional Heterogeneous Agricultural Tri-dimensional Pollution Data Submission Technology}

The platform solves distributed heterogeneous agricultural tri-dimensional pollution data submission in many nodes technology and provides table views and personal control of submission interface fields display function. At first, user can select needed tables at random in the platform and navigation bar will embody user' personal selection and provides pointed interface. Secondly, user can select corresponding field attributes of tables and create personal submission interface. It is convenient for different users to operate different operations. Platform provides data submission item by item and batching submission. (1) In submission data item by item interface, formats of submitted data must be controlled seriously when base administrator submits data to central databases. Platform provides description of submitted attributes data format and clear clue of input error. User can realize submit a small quantity of unpacked data correctly. (2) Batching submission: Platform provides two different batching submission methods: importing from Excel files to central database and importing from local database to central database. By the batching submission module provided by system, users can copy data in local database quickly to central database.

\subsection{Mutual Access Technology of DNDC Model Software and Database}

In the process of DNDC model accumulation software working, users should input relevant data and read in some fixed format text files under some circumstances. All data should be read out from database, so DNDC software is needed to be able to visit database and can read the data. The result data stimulated by DNDC model should be stored in database and DNDC software is required to mutual access with database. Assistant DNDC module can realize DNDC and database mutual access and data transmission. When data is read from database and built up txt files, the platform not 
only shows txt files but also tables and fields in database. So user can decide how to map by himself. When part of result data from DNDC model is stored in database, result files has different formats and store in different formats. After user selects the loading result files, platform will show every data item and field names of tables in database. User can decide how to map data item in result files to fields of tables in the database and read in result data to database. It is convenient to user.

\section{Conclusion}

Combined with our country ecology system actual situation, introducing foreign developed DNDC model and development carbon and nitrogen emission reduction motivation model can help scientists and engineers judge intuitively and make a strategic decision. So we build up a mutual platform in which collect and manage data for DNDC model. For studying agricultural tri-dimension pollution, validation, amendment and operation agricultural tri-dimension pollution carbon and nitrogen emission reduction motivation model needs a lot of data support, so standardization of database is very important. In the platform, database can receive all the collected data from bases administrator. Central system administrator can adjust database according to types of collected data in order to supporting our country actual situation and DNDC model.

\section{Acknowledgements}

This work is supported by the National Science and Technology Major Project of the Ministry of Science and Technology of China (Grant No. 2009ZX03001-019-01), Special fund project for Basic Science Research Business Fee, AIIS(Grant No. 2010-J).

\section{References}

1. Zhang, L.J., Zhu, L.Z.: Agriculture tri-dimensional pollution preventive treatment is strategy requirement in present environment protection. Environmental Protection 05, 36-43 (2007)

2. Qiu, J.J., Wang, L.G., Li, H., Tang, H.J., Li, C.S., Van Ranst, E.: Modeling the impacts of soil organic carbon content of croplands on crop yields in Chin. Agricultural Sciences in China 8(4), 464-471 (2009)

3. Chen, P.Q., Huang, Y., Yu, G.R.: Carbon cycle of terrestrial ecosystem. Science Press, Beijing (2004)

4. Li, H., Wang, L.G., Qiu, J.J.: Aoolication of DNDC model in estimating cropland nitrate leaching. Chinese Journal of Applied Ecology 20(7), 1591-1596 (2009)

5. Huang, M.X., Zhang, S., Zhang, G.L.: Nitrate leaching from a winter wheat summarize rotation in Beijing area. Geographical Research 21(4), 425-433 (2002)

6. Lu, X.Y., Cheng, G.W., Xiao, F.P., Fan, J.H.: Modeling effects of temperature and precipitation on carbon characteristics and GHGs emissions in Abies fabric forest of subalpine. Journal of Environmental Sciences 20, 339-346 (2008) 